\title{
PATTERNS OF SCIATIC NERVE BIFURCATION AND THEIR CLINICAL RELEVANCE
}

\section{Naveena Swargam *1, Shabana Sultana ${ }^{2}$.}

${ }^{{ }_{1}}$ Associate Professor, Department of Anatomy, ESIC Medical College, Sanathnagar, Hyderabad, Telangana State, India.

${ }^{2}$ Tutor, Department of Anatomy, Apollo Institute of Medical Sciences and Research, Hyderabad, Telangana State, India.

\section{ABSTRACT}

Introduction: Sciatic nerve is the largest nerve in the body. It originates from the sacral plexus from L4-S3 roots in the form of two nerve trunks. The Tibial nerve and Common peroneal nerve are encompassed by single epineural sheath and eventually separates. Variations in the level of bifurcation of the sciatic nerve are common and being reported by several authors. The bifurcation into its two major trunks may occur anywhere between the sacral plexus and popliteal space. Significant number of variations in the bifurcation, course, relation and distribution of its branches were encountered in sciatic nerve. These variations may cause nerve compressions under other anatomic structures, resulting in sciatica.

Materials and Methods: 30 lower limbs were dissected in a period of 3 years during the routine dissection to note the relationship between piriformis muscle and sciatic nerve. Only in 2 lower limbs the variation in division of sciatic nerve was observed in the present study.

Results: The literature was reviewed and the results were summarized to compare the incidence with the previous studies. Incidence of this variation in general population needs to be investigated so as to create awareness among surgeons and anaesthetists about the degree and extent of variation in sciatic nerve formation. Complete sciatic nerve blockages will fail even after multiple punctures and attempts if the sciatic nerve is present as separately sheathed bundles until the lower gluteal level.

Conclusion: The aim of this study was to provide and define the level of the sciatic nerve exit, its divisions and its anatomical variations obtained from human cadavers. The availability of assessment of level of bifurcation of sciatic nerve was minimal in the Indian literature so this study was taken to focus a fresh light on the frequency of the level of bifurcation of sciatic nerve. The differences in the exit of two divisions of sciatic nerve are important in clarifying the clinical etiology and treatment of pyriformis syndrome.

KEY WORDS: Sacral plexus, Epineural sheath, Sciatica, Pyriformis syndrome.

Address for Correspondence: Dr. Naveena Swargam, Associate Professor, Department of Anatomy, ESIC Medical College, Sanathnagar, Hyderabad, Telangana State, India.

Ph. No. 09885124371 E-Mail: naveena.swargam@yahoo.com

\section{Access this Article online}

\section{Quick Response code}

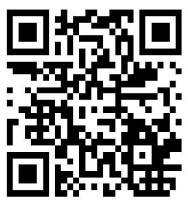

DOI: $10.16965 /$ ijar.2017.123

Web site: International Journal of Anatomy and Research

ISSN 2321-4287

www.ijmhr.org/ijar.htm

Received: 23 Jan 2017

Peer Review: 23 Jan 2017

Revised: None
Accepted: 24 Feb 2017

Published (O): 31 Mar 2017

Published (P): 31 Mar 2017

\section{INTRODUCTION}

Sciatic nerve is the longest and widest nerve in the human body. It is derived from the spinal nerves L4-S3. It contains fibres from both the anterior and posterior divisions of the lumbosacral plexus. It runs from the side of the vertebral column leaves the pelvic cavity through the greater sciatic foramen below the piriformis muscle to enter the gluteal region and descends into the back of 
thigh. Commonly at the apex of popliteal fossa the sciatic nerve bifurcates (85-89\%) into Tibial nerve and Common peroneal nerve. Pain caused by a compression or irritation of the sciatic nerve is called sciatica. The sciatica symptoms include nerve pain, numbness, tingling and weakness. It may include inability to walk depending upon the where the pressure of the sciatic nerve occurs.

Anatomical variations of the relationship between the sciatic nerve and the piriformis muscle were rare. The present study aimed at describing and analysing the sciatic nerve variation in relativity to the statistical analysis. The knowledge of the variation in the level of bifurcation of the sciatic nerve should be kept in consideration while performing surgical exploration. Clinical significance and some relevant previous studies will be presented in this work. A frequent variation in this regard calls for surgeons attention to avoid error in the treatment.

\section{MATERIALS AND METHODS}

30 gluteal regions were dissected in 15 formalin fixed cadavers with no pathology in a period of 3 years irrespective of the sex, during routine dissection for $1^{\text {st }}$ MBBS students, to reveal variations of sciatic nerve. The Gluteus maximus was reflected to explore the piriformis and the sciatic nerve. Following the proper exposure the location of the sciatic nerve, its exit from the pelvis and the level of its division were all recorded. Normally Sciatic nerve bifurcates at the superior angle of popliteal fossa in $80-90 \%$ of individuals.

\section{RESULTS}

Variations in sciatic nerve bifurcation were seen in 2 cadavers. Totally 2 lower limbs showed variation in the division of sciatic nerve (Figures 1 \& 2 ). The right lower limb in one cadaver showed that sciatic nerve divided in the pelvis, common peroneal nerve emerged through the bifid piriformis and tibial nerve below the muscle (Figure 1). Sciatic nerve in the left lower limb of same cadaver divided about $50 \mathrm{~mm}$ above the popliteal crease but below the superior angle of popli- teal fossa. The left lower limb of another cadaver showed that sciatic nerve divided above the ischial spine with the tibial nerve below the prirformis and common peroneal emerged through the piriformis (Figure 2). The Sciatic nerve in the right lower limb of same cadaver divided at the level of the popliteal crease.

Fig. 1: Pelvic division of Right Sciatic nerve.

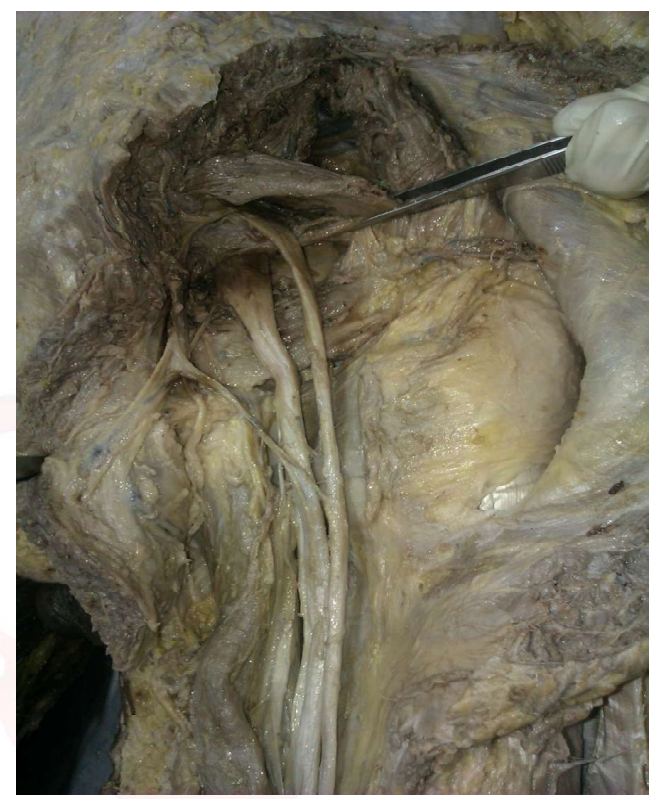

*Right Common peroneal nerve emerging through the piriformis muscle and right Tibial nerve lying deep to the piriformis muscle

Fig. 2: Division of Left Sciatic nerve above the ischial spine.

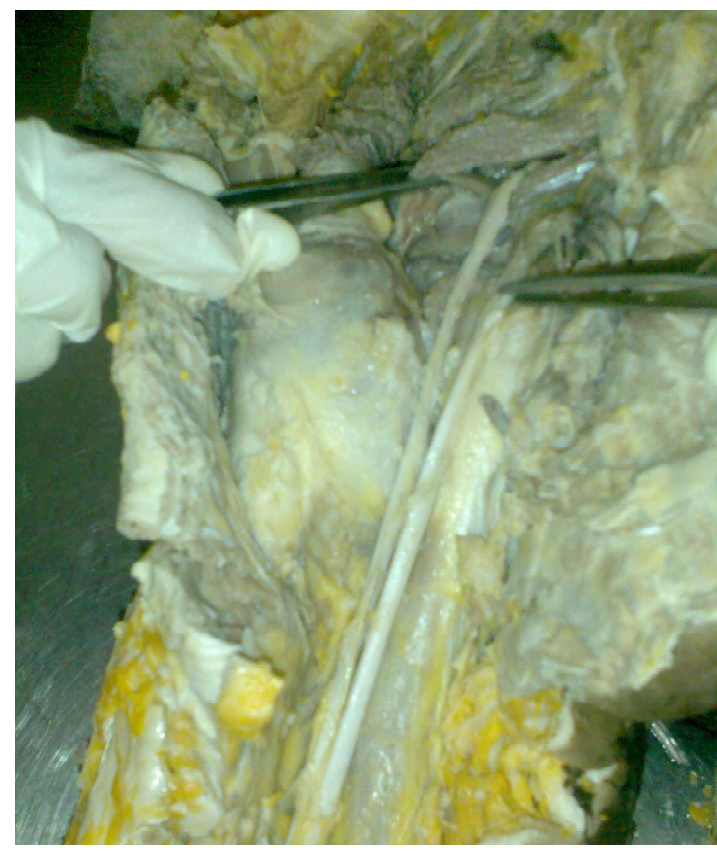

* Left Common peroneal nerve emerging through the piriformis muscle and left Tibial nerve lying deep to the piriformis muscle 
Table 1: Variation in the exit pattern of sciatic nerve.

\begin{tabular}{|c|c|}
\hline & Incidence (\%) \\
\hline Present study & 6.66 \\
\hline Kathe Dinesh Prakash et al. 2014 [6] & 6.67 \\
\hline Moore and Dalley 1999 [7] & 12.2 \\
\hline
\end{tabular}

*Incidence of high division of sciatic nerve with common peroneal piercing the piriformis.

\section{DISCUSSION}

It is unclear whether the anatomical variations in the relationship between the sciatic nerve and the piri-formis muscle are responsible for the pain experienced in piriformis syndrome since asymptomatic patients might have these variations while symptomatic pa-tients might not have them as explained in a study by Kirschner JS et al. (2009)[1]. In fact there is still a tendency to envisage parallel existence of sciatic-piriformis anomalies and piriformis syndrome. Smoll NR. (2010)[2] mentioned in his study that whether the relationship between nerve and muscle is really cause and consequence of the pain remains to be defined. As noted by Vicente EJD et al. (2007) [3] variations in sciatic nerve width and length are not unknown and possibly do not represent an anomaly.

Even in a situation of normal relationship between the sciatic nerve and the piriformis muscle, any condition affecting the muscle (e.g., inflammation or trauma) could indirectly affect the nerve. This idea seems to be particularly supported by the pain relief results achieved when low doses of botulinum toxin are injected into the piriformis muscle of patients with typical signs and symptoms of the piri-formis syndrome according to a study by Yoon SJ et al. (2007)[4]. As described by Stewart JD. (2003)[5] piriformis syndrome is an underdiagnosed cause of glu-teus and leg pain, but according to some authors it is vastly over diagnosed. Variation of bifurcation of sciatic nerve in the present study reviewed and tabulated in comparison with the previous studies by Dr. Kathe Dinesh Prakash et al. (2014)[6] and Moore KL, Dalley AF. (1999)[7] which is shown in Table 1.

The data obtained in the present study did not show any statistically significant difference. The relatively small number of limbs studied in the present work which does not allow for statistical comparisons and conclusions between normal and variant relationships between nerve and muscle. The essence of this study was descriptive, in order to obtain more informa-tion on variations of the sciatic nerve anatomy.

\section{CONCLUSION}

Anatomical variations in the relationship between the sciatic nerve and the piri-formis muscle do not seem to be solely responsible for the piriformis syndrome. High division of sciatic nerve results in sciatica, nerve injury during deep intramuscular injections in gluteal region, failed sciatic nerve block in anaesthesia and injury during posterior hip operations. The knowledge of anatomical variations in the course of sciatic nerve is having great importance to surgeons, orthopedicians and anaesthetist during sciatic block to improve clinical results.

\section{Conflicts of Interests: None}

\section{REFERENCES}

[1]. Kirschner JS, Foye PM, Cole JL. Piriformis syndrome, diagnosis and treatment. Muscle Nerve. 2009;40(1):10-8.

[2]. Smoll NR. Variations of the piriformis and sciatic nerve with clinical consequence: a review. Clin Anat 2010;23(1):8-17.

[3]. Vicente EJD, Viotto MJS, Barbosa CAA, et al. Study on anatomical relationships and variations be-tween the sciatic nerve and piriform muscle. Rev Bras Fisioter 2007;11(3):227-32.

[4]. Yoon SJ, Ho J, Kang HY, et al. Low-dose botulinum toxin type $A$ for the treatment of refractory piriformis syndrome. Pharmacotherapy. 2007;27(5):65765.

[5]. Stewart JD. The piriformis syndrome is over diag-nosed. Muscle Nerve 2003;28(5):644-6.

[6]. Dr. Kathe Dinesh Prakash et al. Study of anatomical variations of the sciatic nerve and its importance to clinicians and anaesthetist. International Journal of Current Research. 2014;6(7):7518-7521.

[7]. Moore KL, Dalley AF. Clinical Oriented Anatomy, 4th edition, Baltimore Lippincott Williams and Wilkins: 1999;558.

How to cite this article: Naveena Swargam, Shabana Sultana. PATTERNS OF SCIATIC NERVE BIFURCATION AND THEIR CLINICAL RELEVANCE. Int J Anat Res 2017;5(1):3622-3624. DOI: 10.16965/ijar.2017.123 